\title{
Cross-estimation of Soil Moisture Using Thermal Infrared Images with Different Resolutions
}

\author{
Wei-Ling $\mathrm{Hsu}^{1}$ and Kuan-Tsung Chang ${ }^{2 *}$ \\ ${ }^{1}$ Huaian Key Laboratory of Geographic Information Technology and Applications, \\ School of Urban and Environmental Science, Huaiyin Normal University, \\ No. 111, Changjang W. Rd., Huai'an City, Jiangsu, P. R. China \\ ${ }^{2}$ Department of Civil Engineering and Environmental Informatics, \\ Ming Hsin University of Science and Technology, No. 1, Xinxing Rd., Xinfeng Hsinchu 30401, Taiwan
}

(Received May 29, 2018; accepted October 11, 2018)

Keywords: soil characteristics, remote sensing, soil moisture, thermal sensor

Land use, land cover, and carbon dioxide emission reduction have been crucial topics among scholars considering the prevention of global warming in recent years. The application of satellite remote sensing technology and aerial images using unmanned aerial vehicles (UAV) equipped with sensors has become common in large-scale environmental monitoring. In this experimental study, we used satellite images and aerial images to quantitatively analyze the effect of soil moisture on the regional thermal environment. Empirical equations of soil linearity, temperature vegetation dryness index (TVDI), and apparent thermal inertia (ATI), among other parameters, were compared. The study area was farmland in Hsinchu, Taiwan. Land surface temperature (LST) was measured using a multiplatform infrared sensor, and actual soil moisture was measured using a soil moisture meter on-site. The parameters for estimating the moisture of surface soil (i.e., land surface temperature, normalized difference vegetation index (NDVI), images of thermal inertia, and empirical equations of soil moisture) were obtained using a geographical information system (GIS). Finally, the soil moisture estimations were compared with on-site soil moisture measurements. Regression analysis was employed to compare the correlation between satellite and aerial data to establish a surface-soil moisture estimation model in areas of different land covers. The results indicated that the soil moisture estimated using the empirical equation of soil linearity was closest to that in actual measurements, followed by the estimations based on the temperature vegetation dryness index and apparent thermal inertia.

\section{Introduction}

Recently, applications of satellite remote sensing technology and aerial images using unmanned aerial vehicles (UAV) have facilitated the development of environment-monitoring technologies. Such technologies enable the efficient collection of large-scale monitoring data for different time points. Changes in land use and land cover are critical driving forces

*Corresponding author: e-mail: ktchang1216@gmail.com

https://doi.org/10.18494/SAM.2019.2090 
behind variations in the atmosphere, climate, and ecosystems. ${ }^{(1)}$ Sensors used in remote sensing technology acquire surface information by intercepting electromagnetic waves. These electromagnetic waves are surface radiation, artificially launched electromagnetic waves, or radiation emitted by the sun. When incident electromagnetic radiation hits a surface object, reflection, scattering, absorption, and transmission occur. A remote sensing system can detect surface reflection spectra from which surface characteristics can be elucidated. In recent studies, spectra covering various wavelengths and obtained from satellite images have been employed as monitoring parameters. Spectral data generated by on-site sampling have been compared with data indicators to confirm the accuracy of surface monitoring technologies. ${ }^{(2)}$ Researchers have measured and analyzed the electromagnetic spectra reflected or emitted from land surfaces and crop canopies. These spectra can be classified as visible light (400-700 nm), near-infrared light $(700-1300 \mathrm{~nm})$, mid-infrared light (1300-2500 nm), far-infrared light (8-14 $\mu \mathrm{m})$, or microwave $(1 \mathrm{~mm}-1 \mathrm{~m})$ spectra. Among the associated wavelength bands, visible, near- and far-infrared light are most frequently used; however, the applications of microwave light are also prominent. ${ }^{(3-5)}$ Remote sensing technology employs aerial images obtained from expensive and complicated satellites, but the technology saves the time and human resources that would be required to conduct on-site monitoring. ${ }^{(6,7)}$ Among the various technologies used to monitor changes in land cover, light detection and ranging are effective tools for obtaining large-bandwidth spectra and spatial data. ${ }^{(8)}$ In this study, we compared data obtained from satellite images (from satellite Landsat 8) and data (i.e., aerial images, infrared inertial images, and multispectral images) obtained from a UAV with on-site measurement data. Numerous parameters were employed to estimate the moisture in surface soil using empirical equations for soil moisture. Generated using a geographical information system (GIS), these parameters were the temperature vegetation dryness index (TVDI), normalized difference vegetation index (NDVI), land surface temperature (LST), and apparent thermal inertia (ATI). Finally, the estimated soil moisture was compared with the actual soil moisture measured on-site. The data obtained using the two technologies were assessed by regression analysis. In this study, we adopted various vehicles, including satellite images and various thermal imaging sensors with different resolutions carried by a UAV, to estimate soil moisture. The results were compared with those of on-site measurements and empirical equations, the TVDI, and ATI to evaluate their applicability.

\section{Materials and Methods}

Three remote sensing methods can be used to estimate soil moisture: direct measurement, indirect measurement (through topographic wetness indices), ${ }^{(9)}$ and surrogate measures (through the characteristics of vegetation reflectance). ${ }^{(10)}$ According to the results of previous studies, optimal surface-soil moisture estimations can be obtained by direct measurement. Direct measurement can be conducted using passive microwaves, ${ }^{(1)}$ active microwaves, ${ }^{(12)}$ thermal infrared (TIR), ${ }^{(13)}$ and visible short-wave infrared. ${ }^{(14)}$ In the present study, we used multiplatform infrared images to estimate the moisture in surface soil. Data obtained through direct measurement were compared with those measured using a soil moisture meter. The results and characteristics of the direct measurement were comprehensively determined. The 
experiment was conducted on farmland in Hsinchu County, Taiwan. Satellite and aerial images (taken by a UAV) were collected. A GIS was used to calculate and analyze image data. The empirical equation of soil moisture was imported into the GIS to establish a mathematical relationship. The results of regression analysis were compared to develop and verify the relationships among the data from satellite images, UAV-obtained thermal inertial images, and soil moisture measurements.

\subsection{Thermal inertia}

Soil thermal inertia is an intrinsic factor that reflects temperature changes in surface soil and is closely related to soil moisture. In this study, we used the diurnal temperature range of LST to estimate the moisture in surface soil. Additionally, a thermal imaging camera on a UAV was employed to capture TIR images over a range of temperatures during the experiment to determine the thermal inertia. For a given soil medium, the larger the medium's thermal inertia, the more slowly its temperature can change. In this study, thermal inertia was calculated on the basis of the expression of ATI. ${ }^{(15)}$

\subsection{Soil moisture indices}

Conventional soil moisture monitoring methods are not only time-consuming and labor intensive but also involve few measurement points and exhibit poor representativeness. Therefore, these conventional methods cannot meet the requirements for wide-ranging and dynamic monitoring in the field. Remote sensing measurement technologies can satisfy these needs. Compared with conventional monitoring technologies, remote sensing provides timely and consistent large-scale monitoring data. The monitoring results are objective, and the technical costs are low. Remote sensing has thus received considerable attention. The temperature difference between daytime and nighttime can be obtained by calculating soil moisture based on the TIR band. According to the principle of thermal inertia, a smaller temperature difference indicates higher soil moisture. However, the exact relationship varies according to the soil composition and texture. The reflectance of each wavelength band has a different effect on the soil; therefore, the indices of the detected band are differently related to soil moisture. The indices related to soil moisture are as follows.

1. The TVDI is an index describing the soil moisture in areas covered by vegetation and is based on optical and TIR data. ${ }^{(16)}$

2. The NDVI ${ }^{(17)}$ is defined as the difference between near-infrared reflectance and red reflectance divided by the sum of near-infrared reflectance and red reflectance. ${ }^{(18)}$

3. LST is the temperature of the heat released after the land surface has absorbed the heat from the sun. ${ }^{(19)}$

\subsection{Experimental procedure}

First, images obtained using the Landsat 8 satellite were employed; ${ }^{(20)}$ these images were provided by the United States Geological Survey (USGS). After radiation correction and 
ground temperature calculation, empirical equations for soil moisture and TVDI were used to estimate the soil moisture in the study area. The results were verified against actual soil moisture measurements. Second, a UAV carrying a thermal imaging camera was employed to take aerial images of the study area in the daytime and nighttime. Simultaneously, on-site soil moisture was measured. Thermal inertia was calculated using image data obtained with the UAV; the soil moisture could then be estimated from the thermal inertia values derived from the surface temperature during the daytime and nighttime. The empirical equation of soil moisture and the TVDI were also used on the images from the UAV. The soil moisture in the study area was estimated, and the estimated results were compared with the actual soil moisture measurements. The following briefly describes the experimental procedure.

1. Objective: To obtain information-including on-site soil moisture, thermal inertia, and satellite images data - for farmland in Hsinchu County in Taiwan.

2. Experimental method: A portable soil moisture meter (HOBO-U30) and thermal imaging camera (on a UAV) were used; satellite images of the study area were downloaded from the USGS website.

3. Measurements in the study area: Four sensors were connected to the soil moisture meter and inserted into the soil to measure soil moisture over a period of several minutes. A GPS device recorded the measurement locations, and a UAV took thermal images of the study area.

4. Analysis of experimental data: Data from the satellite- and UAV-obtained images were inserted into the empirical equations for soil moisture, TVDI, and thermal inertia. Subsequently, regression analysis was performed, and the results were compared with the actual soil moisture data.

5. Analysis of experimental results: The differences between the indices calculated from the different data types and the regression curves were analyzed.

\section{Results}

The study area, covering approximately 40 hectares, was the farmland area along the Touqian River in Hsinchu County. The experiment was conducted in September 2017. At the time of the experiment, the majority of the farmland lay fallow, and the open land was suitable for sampling and measuring soil moisture.

\subsection{Satellite image analysis}

The reference equations and values were imported into the raster calculator of ArcGIS. The aforementioned equations were inserted into the function table, and the calculated cartographic data were subsequently imported into the calculator. The satellite images were corrected and combined in accordance with the requirements of the estimation methods employed. The calculated and combined images of LST and NDVI maps are presented in Figs. 1 and 2, respectively.

The following equations were employed to calculate the radiometric calibration temperature of the satellite sensor (BT), 


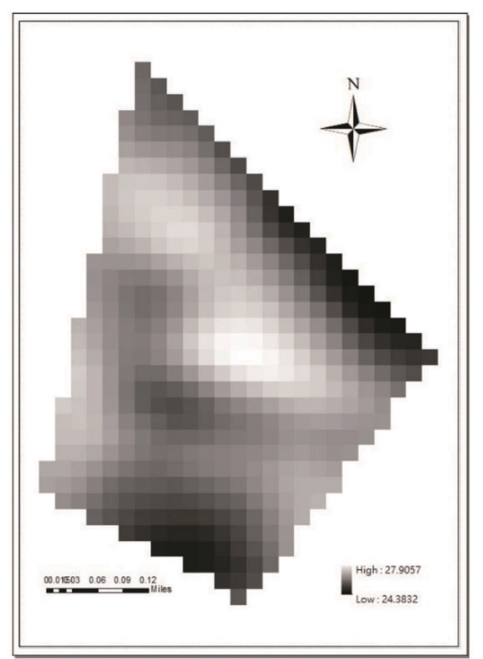

Fig. 1. LST map of satellite image.

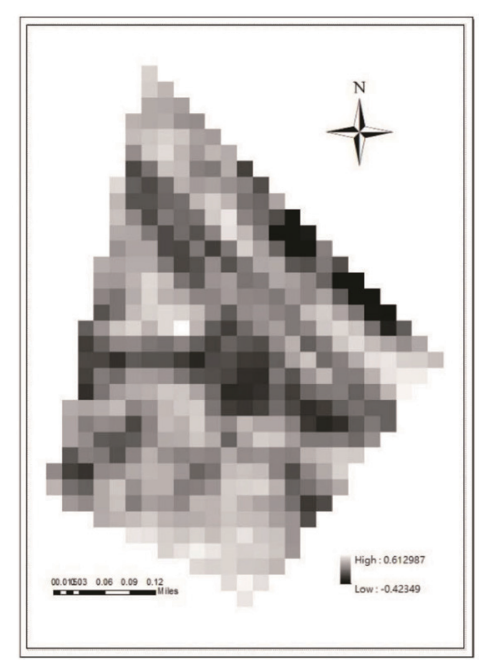

Fig. 2. NDVI map of satellite image.

$$
B T=\frac{K 2}{\ln \left(\frac{K 1}{L \lambda}+1\right)},
$$

the LST,

$$
L S T=\frac{B T}{1+\frac{\lambda \times B T}{\rho} \times \ln \varepsilon},
$$

the ground radiological survey,

$$
\begin{aligned}
P_{v} & =\frac{\left(N D V I_{j r}-N D V I_{\text {min }}\right)^{2}}{\left(N D V I_{\max }-N D V I_{\text {min }}\right)} \\
\varepsilon & =0.004 \times P_{v}+0.986,
\end{aligned}
$$

the NDVI,

$$
N D V I=\frac{\operatorname{Red}-N I R}{\operatorname{Red}+N I R},
$$

and the TVDI,

$$
T V D I=\frac{L S T_{s}-L S T_{\min }}{L S T_{\max }-L S T_{\min }} .
$$


Soil linearity indicates the degree of correlation between the reflectances of red light and near-infrared light. A change in humidity exhibits a strong effect on these two bands. Therefore, the reflectances of the wettest and driest soils in the study area were analyzed, as presented in Fig. 3.

The TVDI from satellite images was used to estimate the moisture of surface soil. Specifically, the satellite image data were input into Eqs. (1) and (2) to derive LST. Subsequently, LST was substituted into Eq. (6) to obtain the TVDI. The data obtained from the TVDI calculations were values for dry conditions, which negatively correlated with the soil moisture. The TVDI estimated from the satellite images is plotted in Fig. 4.

\subsection{UAV-obtained image analysis}

The soil linearities of the wettest and driest surface soil reflectances of red light and near-infrared light were obtained from the aerial images. The two wavebands substantially correlated, as denoted in Fig. 5.

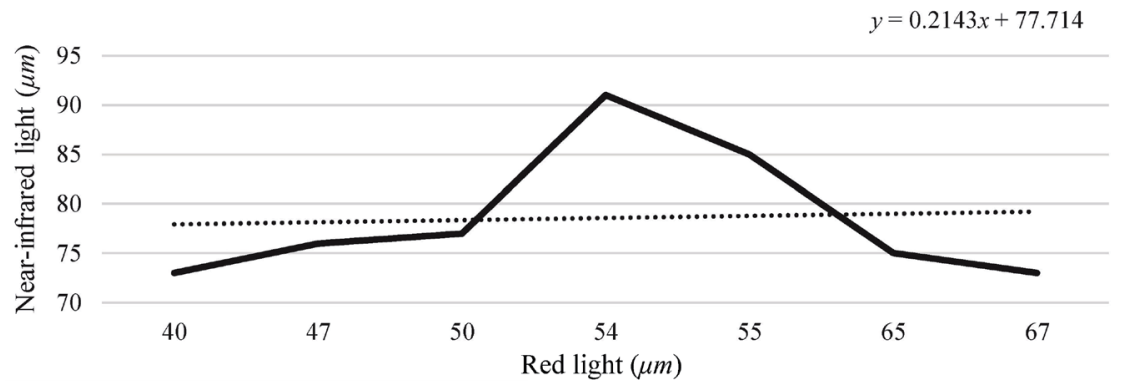

Fig. 3. Soil linearity.

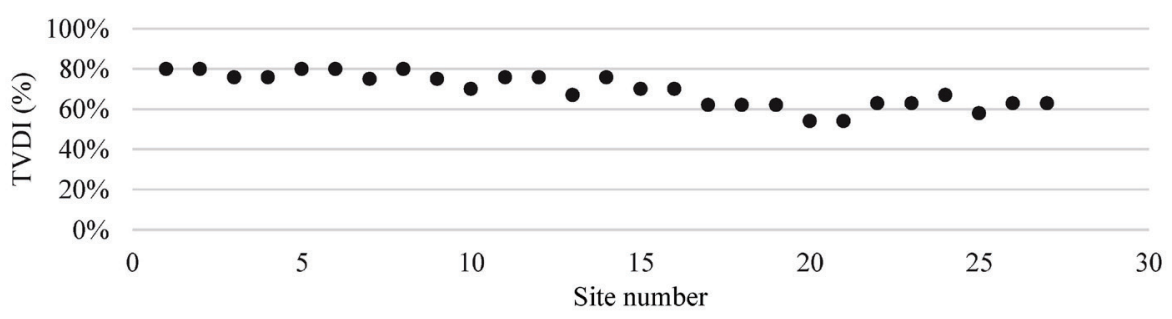

Fig. 4. Soil moisture in surface soil, estimated from satellite images.

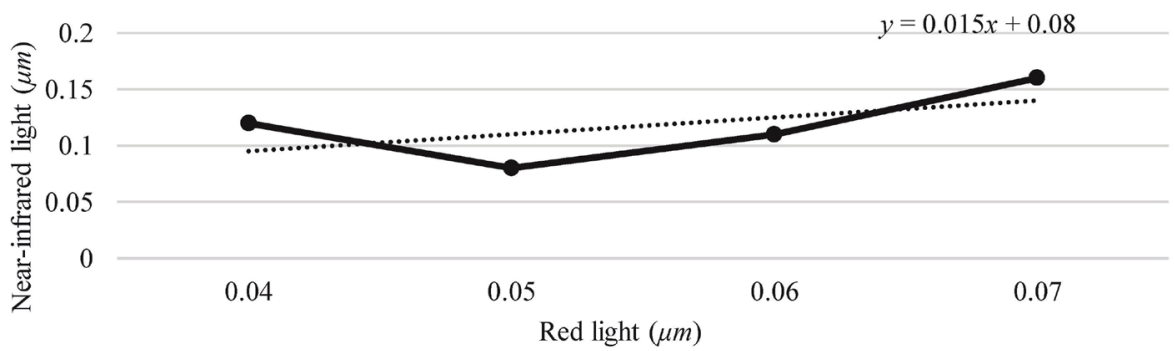

Fig. 5. Soil linearity (estimated from aerial images). 
Referring to the measured soil moisture, the wet and dry soil reflectances of red and nearinfrared light were determined. Reflectance was used to obtain the equation of soil linearity. Subsequently, a straight line passing through the wet soil data and perpendicular to the soil line was obtained. The soil moisture estimated from the soil linearity is plotted in Fig. 6.

The thermal images taken by the UAV comprised images from the times of the lowest (dawn) and highest (noon) temperatures in one day. These thermal images were incorporated into one image representing the average temperature of the day by using ArcGIS, and the TVDI of the image was then calculated. The cell statistics function of ArcGIS was employed to average the daytime and nighttime images. After an average image was obtained, the effect of the TVDI on the moisture in surface soil was calculated. The TVDIs corresponding to measurement points are presented in Fig. 7.

\section{Discussion}

Regression analysis was conducted on soil moisture data obtained from Landsat 8 satellite images, multispectral images captured by a UAV, and actual field measurement data. Regression analysis is often used in data interpretation and prediction. For data interpretation, sample data can be employed to calculate regression equations, and these equations describe the effect of each independent variable on the dependent variable. This method of analysis was applied to statistically interpret the moisture in surface soil. Figure 8 denotes the soil moisture values measured using the soil moisture meter. The obtained data were compared with those estimated using numerous images and equations.

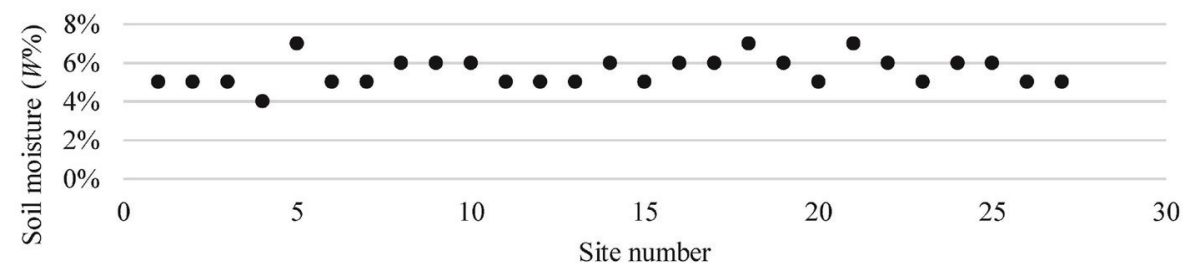

Fig. 6. Soil moisture estimated from UAV thermal images using the soil linearity method.

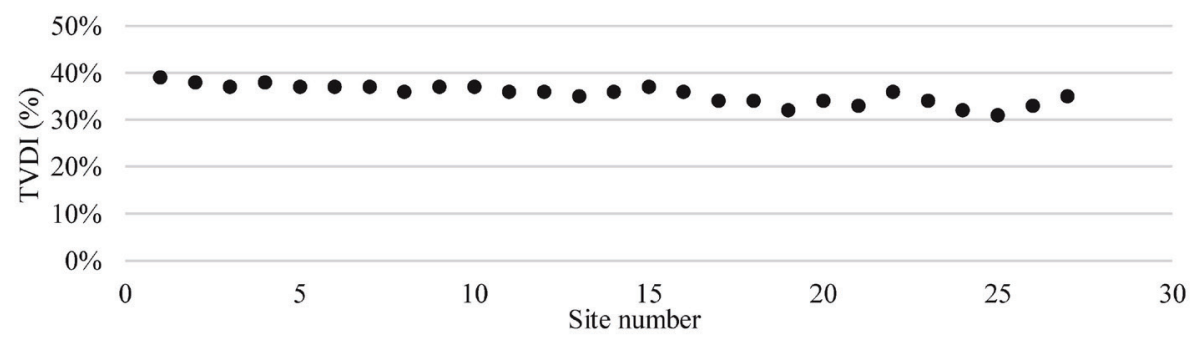

Fig. 7. TVDIs estimated from the aerial images. 


\subsection{Equation of soil linearity}

Here, the soil moisture calculated from satellite images, aerial images, and actual soil moisture data is discussed. Regression analysis was performed to identify the variation in each curve. Figures 9-12 display the results of the moisture in surface soil and the differences in soil moisture between the satellite and aerial images. The $R^{2}$ of the satellite image plot was 0.3 , whereas that of the aerial image plot was 0.016 .

\subsection{TVDI}

In this section, we discuss the TVDI of soil moisture obtained from the satellite images, aerial images, and on-site measurement data. Figures 13 and 14 show these TVDIs. The $R^{2}$ of the satellite image plot was 0.71 , whereas that of the aerial image plot was 0.42 .

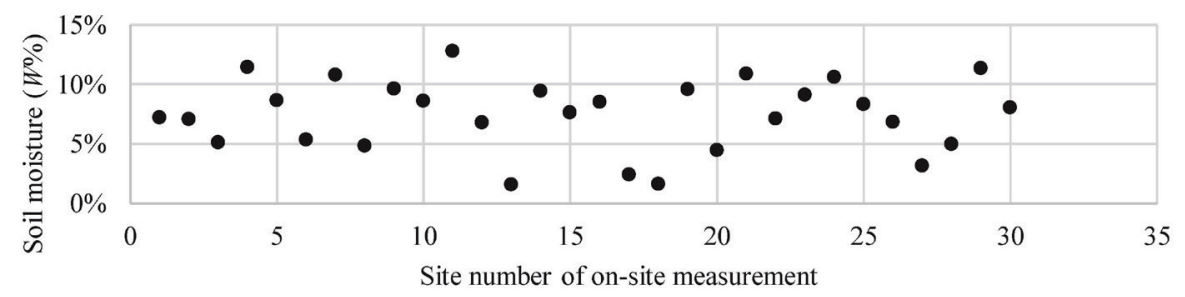

Fig. 8. Soil moisture measured on-site.

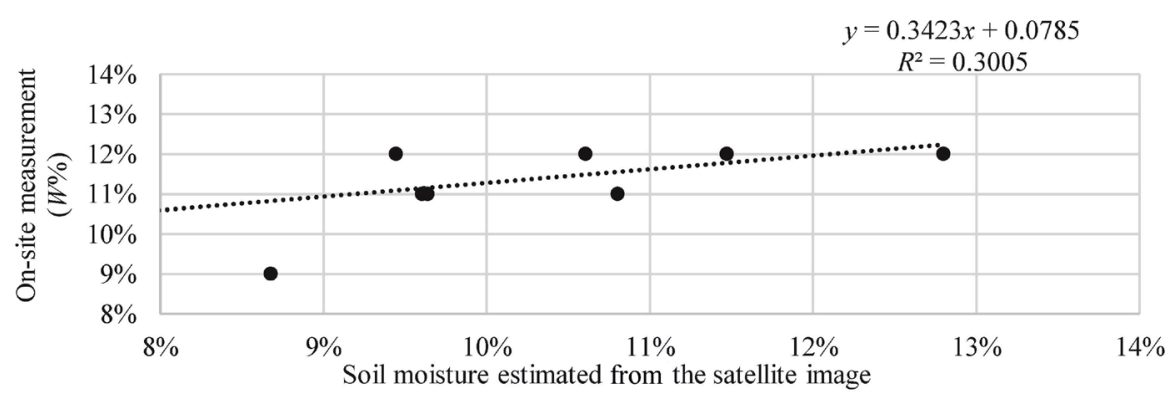

Fig. 9. Soil moisture estimated from satellite image and measured on-site.

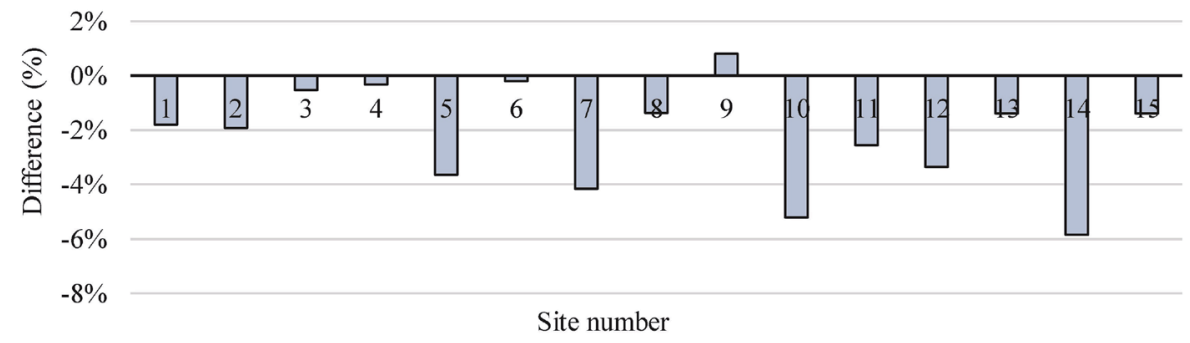

Fig. 10. (Color online) Difference between soil moisture estimated from satellite image and measured on-site. 


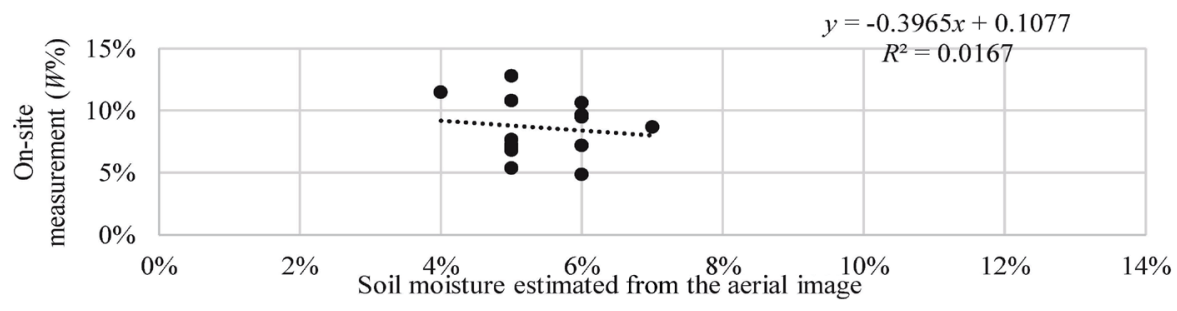

Fig. 11. Soil moisture estimated from aerial image and measured on-site.

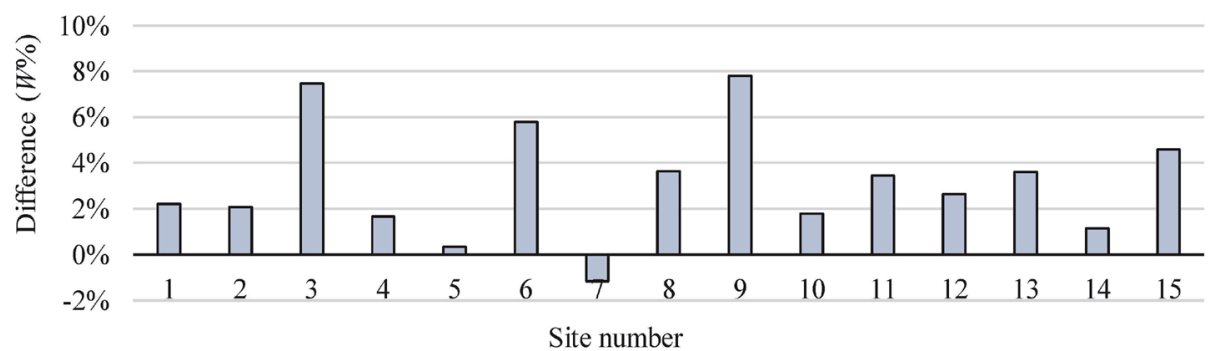

Fig. 12. (Color online) Difference between soil moisture estimated from aerial image and measured on-site.

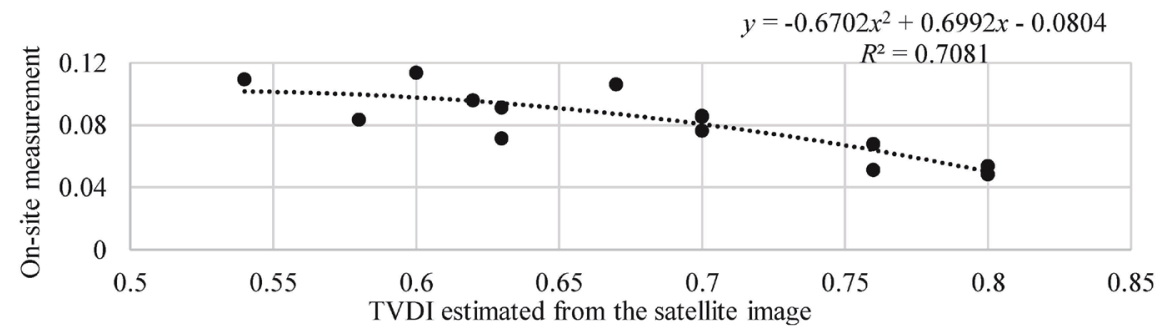

Fig. 13. TVDI of soil moisture estimated from satellite image and measured on-site.

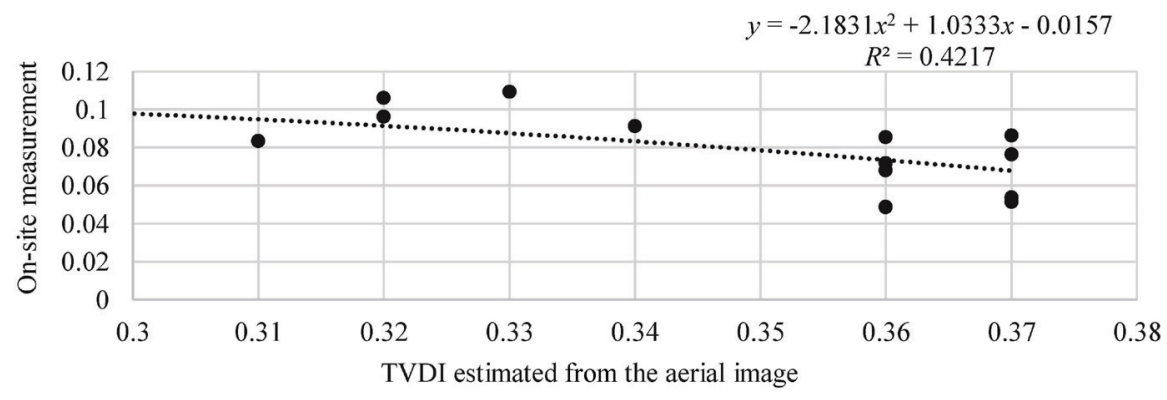

Fig. 14. TVDI of soil moisture estimated from aerial image and measured on-site. 


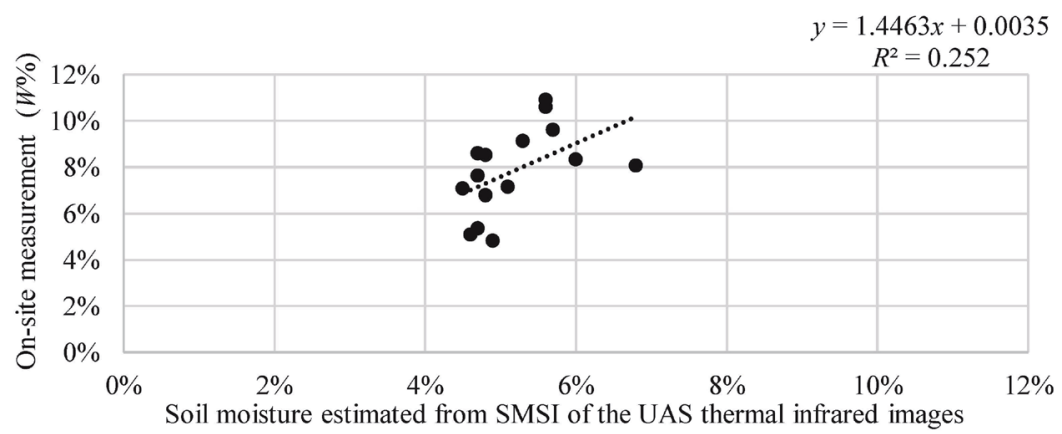

Fig. 15. Soil moisture saturation index (SMSI) and soil moisture measured on-site.

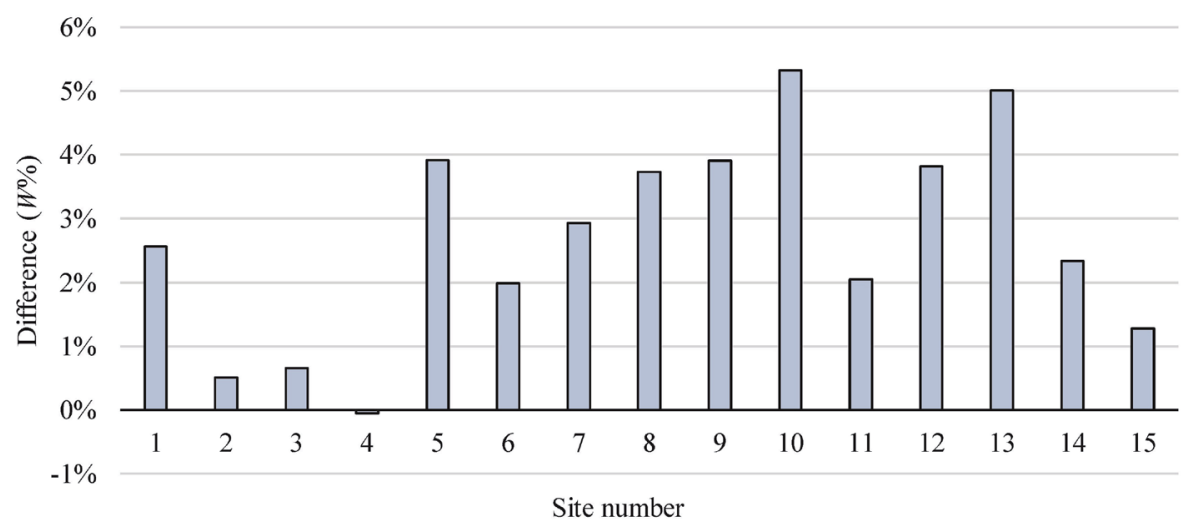

Fig. 16. (Color online) Differences between SMSI and soil moisture measured on-site.

\subsection{Thermal inertia}

Thermal inertia data were derived from the UAV TIR images (the lowest temperature was obtained at 4:00-5:00 AM, and the highest temperature at 1:00-2:00 PM in one day). The satellite flies over Taiwan once per day. In this study, regression analysis of the thermal inertia data was conducted, and variations in regression curves were examined. The results of the regression analysis were used to calculate soil moisture, and the results are presented in Figs. 15 and 16.

\section{Conclusion}

In this study, we estimated the moisture in surface soil using satellite images and aerial images captured with a thermal imaging camera and multispectral camera placed on a UAV. The soil moisture was estimated from the two types of images and compared with that measured using a soil moisture meter in the study area. Measurement sites were positioned using a GPS. The results of the regression analysis revealed that the soil moisture calculated from the TVDI was more favorable than that calculated from the thermal inertia and empirical equations. The experimental results indicated that the soil moisture estimated using the model of the TVDI was closest to the actual soil moisture measured on-site; the value estimated from 
the empirical equation of soil linearity and soil moisture was the second-most-accurate result. The study area primarily comprised paddies or dry fields, and the soil moisture estimated from satellite images was subject to errors owing to plant shade. The relationship between the TVDI and soil moisture was estimated on the basis of the NDVI and LST, where the NDVI was an indicator of vegetation change. Therefore, the effect of plant shade was considerably reduced in these calculations. The LST greatly differs among areas according to factors such as those of vegetation, water, and wet soil. Dense vegetation, water, and wet soil are found in relatively humid regions. Accordingly, in the study area, temperatures were relatively low. The surface soil in the study area was rough, and the images captured from the UAV had high resolution. Therefore, the scattering of light by soil during the reading and receiving of signals affected the accuracy of the calculation. The satellite images exhibited large pixels; therefore, calculations made using satellite data involved fewer errors due to the poorer image quality. In future studies, researchers should collect and compare additional on-site soil moisture data from different time points and a large-scale study area by using satellite and aerial images to increase the accuracy of soil moisture estimation.

\section{Acknowledgments}

We are grateful to the participants of the research team. We thank the experts and scholars for reviewing, editing, and providing valuable comments on the manuscript.

\section{References}

1 K. T. Chang, G. W. Lee, L. S. Liang, J. K. Liu, and F. C. Yu: 2017 IEEE Int. Geoscience and Remote Sensing Symp. (IGARSS) (IEEE, 2017) 6275. https://doi.org/10.1109/IGARSS.2017.8128441

2 P.-H. Hsu: Sen. Mater. 28 (2016) 455. https://doi.org/10.18494/SAM.2016.1301

3 T. H. Demetriades-Shah, M. D. Steven, and J. A. Clark: Remote Sens. Environ. 33 (1990) 55. https://doi. org/10.1016/0034-4257(90)90055-Q

4 M. Steven and J. A. Clark: Applications of Remote Sensing in Agriculture (Elsevier, London, 2013). https:// www.elsevier.com/books/applications-of-remote-sensing-in-agriculture/steven/978-0-408-04767-8

5 C. J. Johannsen, P. G. Carter, P. R. Willis, E. Owubah, B. Erickson, K. Ross, and N. Targulian: Precision Agriculture (American Society of Agronomy, Crop Science Society of America, Soil Science Society of America, Madison, WI, 1999) p. 1413. https://doi.org/10.2134/1999.precisionagproc4.c43b

6 E. C. Barrett: Introduction to Environmental Remote Sensing (Routledge, New York, 2013). https://www. routledge.com/Introduction-to-Environmental-Remote-Sensing/Barrett/p/book/9780748740062

7 T. Lillesand, R. W. Kiefer, and J. Chipman: Remote Sensing and Image Interpretation (Wiley, NJ, USA, 2014). https://www.wiley.com/en-us/Remote+Sensing+and+Image+Interpretation\%2C+7th+Edition-p-9781118343289

8 K. T. Chang, F. C. Yu, Y. Chang, J. T. Hwang, J. K. Liu, W. C. Hsu, and T. Y. Shih: Terr. Atmos. Ocean. Sci. 26 (2015) 169. https://doi.org/10.3319/TAO.2014.12.02.02(EOSI)

9 P. C. Dubois, J. Van Zyl, and T. Engman: IEEE Trans. Geosci. Remote Sens. 33 (1995) 915. https://doi. org $/ 10.1109 / 36.406677$

10 M. F. Noomen, A. K. Skidmore, F. D. Van der Meer, and H. H. Prins: Remote Sens. Environ. 105 (2006) 262. https://doi.org/10.1016/j.rse.2006.07.009

11 A. K. Sahoo, P. R. Houser, C. Ferguson, E. F. Wood, P. A. Dirmeyer, and M. Kafatos: Remote Sens. Environ. 112 (2008) 3142. https://doi.org/10.1016/j.rse.2008.03.007

12 N. Pierdicca, L. Pulvirenti, and C. Bignami: Remote Sens. Environ. 114 (2010) 440. https://doi.org/10.1016/ j.rse.2009.10.001

13 M. Minacapilli, M. Iovino, and F. Blanda: J. Hydrol. 379 (2009) 229. https://doi.org/10.1016/j.jhydrol.2009.09.055

14 Z. Lihua, L. Minzan, S. Jianying, T. Ning, and Z. Xijie: Proc. 2005 IEEE Int. Geoscience and Remote Sensing Symp., 2005. IGARSS’05. 6 (2005) 4516. https://doi.org/10.1109/IGARSS.2005.1525925 
15 S. G. Lei, Z. F. Bian, J. L. Daniels, and D. L. Liu: Trans. Nonferrous Met. Soc. China 24 (2014) 1866. https:// doi.org/10.1016/S1003-6326(14)63265-9

16 I. Sandholt, K. Rasmussen, and J. Andersen: Remote Sens. Environ. 79 (2002) 213. https://doi.org/10.1016/ S0034-4257(01)00274-7

17 J. W. Rouse, Jr., R. Haas, J. Schell, and D. Deering: NASA Goddard Space Flight Center 3d ERTS-1 Symp., 1 Sect. A (1973) 309. https://ntrs.nasa.gov/search.jsp?R=19740022614

18 C. D. Elvidge and Z. Chen: Remote Sens. Environ. 54 (1995) 38. https://doi.org/10.1016/0034-4257(95)00132-K

19 Q. Weng, D. Lu, and J. Schubring: Remote Sens. Environ. 89 (2004) 467. https://doi.org/10.1016/j.rse.2003.11.005

20 USGS: USGS-Earth Explorer. https://earthexplorer.usgs.gov/

\section{About the Authors}

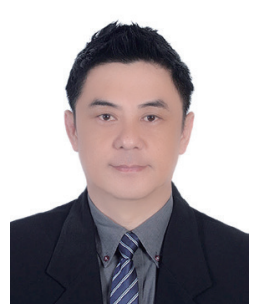

Wei-Ling Hsu received his Ph.D. in 2014 from the Department of Civil Engineering and his Master's degree in architecture in 2009 from Chung Hua University in Taiwan. From 2009 to 2017, he served as an adjunct assistant professor at Chung Hua University and Minghsin University of Science and Technology. He is currently serving at the Huaian Key Laboratory of Geographic Information Technology and Applications, School of Urban and Environmental Science, Huaiyin Normal University, China. His research expertise includes the application of satellites and unmanned aerial vehicles in ground-level environmental monitoring, urban traffic, urban and rural planning, and the incorporation of data analysis in fuzzy multiple criteria decision making for cities and environments.

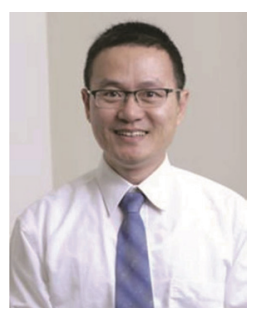

Kuan-Tsung Chang received his B.S. degree from National Cheng Kung University in 1980 and his Ph.D. degree from National Chiao Tung University (NCTU), HsinChu, Taiwan, in 1998. From 2000, he served as an assistant professor at Ming-hsin University of Science and Technology (MUST). He is currently an associate professor and chairman of Civil Engineering and Environmental Informatics at MUST. He conducted several research and development projects in the areas of landslide feature or linear object extraction, hyperspectral image analysis, and pavement capacity measurement using a 3D Laser Scanner. His major research interests are in the fields of environmental remote sensing, geospatial information extraction, engineering surveying, and spatial objects analysis. 